www.nature.com/clinicalpractice/uro

\title{
Single-port surgery for donor nephrectomy: a new era in laparoscopic surgery?
}

Mahesh Desai

The concept of single-port urologic procedures has evoked great interest among urologists. Initial studies of the procedure suggest that laparoendoscopic single-site surgery (LESS) for donor nephrectomy may have the potential to be a future gold-standard procedure (Marescaux J et al. [2007] Arch Surg 142: 823). Clinical outcomes for this technique could be appealing, particularly for kidney donors; single-port surgery is a minimally invasive procedure compared with standard laparoscopic or open nephrectomy. Furthermore, this type of surgery might be safer than traditional methods. However, several issues need to be assessed before single-port surgery can be widely used.

Firstly, kidney retrieval is the most arduous of the steps in single-port procedures. One study describes graft retrieval with a preplaced bag (Gill IS et al. [2008] J Urol 180: 630-641). However, this method makes the procedure complex, and there is a risk of increasing retrieval time. One way around this might be to retrieve the graft by hand after removing the port and extending the incision by $2 \mathrm{~cm}$.

Secondly, instruments that are currently used for single-port surgery are still at their developmental stages, which will result in a steep learning curve for surgeons. The LESS procedure typically requires longer instruments compared with standard laparoscopic procedures, or specialized articulated instruments with a
The main

advantages

of single-site

surgery are

the reduced

number of

incisions

needed, and

the potential for

reduced pain

and reduced

hospital stays

M Desai is Chairman of the Department of Urology at Muliibhai Patel Urological

Hospital, India.

Competing interests

The author declared no

competing interests.

www.nature.com/clinicalpractice doi:10.1038/ncpuro1278 curved shaft to facilitate dissection. In addition, digital laparoscopes with an integrated camera head reduce cluttering of instruments during surgery. However, it is essential that surgical teams have adequate experience and expertise to operate and maintain these new instruments, and it is particularly important that surgeons with substantial experience of routine laparoscopic donor nephrectomy undertake LESS donor nephrectomy.

The main advantages of single-site surgery are the reduced number of incisions needed, and the potential for reduced pain and reduced hospital stays. LESS for donor nephrectomy has the added benefit that it can be performed via the umbilical port, thereby minimizing invasive surgery. LESS via the umbilical port does have an unusual viewing angle, which surgeons must become accustomed to, and proper patient selection and prior planning are essential. Prerequisites for LESS donor nephrectomy could include patient BMI less than $25 \mathrm{~kg} / \mathrm{m}^{2}$ and height less than $175 \mathrm{~cm}$ in initial cases of single vessel grafts, a surgeon with adequate expertise in multiport surgery, and an oriented surgical team for assistance.

Single-port surgery clearly has several advantages, and current evidence is encouraging, but there are several issues that still need to be carefully assessed in multicenter, randomized studies. 